\title{
Professor Yanghee Woo: globalization of surgical innovation for stomach cancer treatment-a long but exciting road
}

Submitted Sep 03, 2019. Accepted for publication Sep 26, 2019.

doi: 10.21037/jgo.2019.09.14

View this article at: http://dx.doi.org/10.21037/jgo.2019.09.14

\section{Editor's note}

During August $17-18$, the $1^{\text {st }}$ CIMS International medical summit 2019 was held successfully in Beijing, China with the focus on current hot topics in Obstetrics, lung cancer, gastrointestinal cancers, proton beam therapy, Neurooncology, neurosurgery and reconstructive plastic surgery. During the conference, we were fortunately to have the opportunity to talk to Prof. Yanghee Woo, a female surgeon scientist from City of Hope Comprehensive Cancer Center who shared her perspective on important components of multimodality management strategies for gastric cancer (GC) (Figure 1).

\section{Expert's introduction}

Yanghee Woo, MD (Figure 2), is a surgeon scientist specially trained and internationally recognized in robotic surgery and GC. She has particular expertise in minimally invasive approaches to complex procedures such as D2 lymphadenectomy for locally advanced GC. Dr. Woo offers her patients completely laparoscopic and robotic surgeries (for stomach and pancreas tumors) with optimal oncologic outcomes while preserving patients' quality of life. As a researcher, she investigates biomarkers for diagnosis and treatment of gastrointestinal cancers, innovative surgical approaches and novel therapeutic strategies to improve cancer patient outcomes. Highly published and sought out for her unique dedication to GC care in the United States, Dr. Woo has recently joined the Board of Editors of fournal of Gastrointestinal Oncology.

\section{Interview}

FGO: Your speech is about the multimodality management strategies for GC. Could you please summarize the main points to the experts who are not at the conference?

Prof. Woo: GC treatment is evolving quickly as new scientific discoveries and technologic advancements enter the field of cancer care. Globalization of clinical trials and standardization of treatment paradigms, GC patient outcomes are improving but we still have a long ways to go to eliminate this terrible disease that is the third leading cancer killer in the world. Treatment options for GC include radical surgery, chemotherapy, radiation therapy, and new targeted agents such as trastuzumab and immune check point inhibitors such pembrolizamab. Of these, only surgery or radical resection is curative.

The key to GC cure is prevention and early detection but most GC are diagnosed in advanced stages where multimodality therapy is a must to decrease recurrence and increase GC free life. The treatment of early GC requires proper and accurate clinical staging and timely resection. The key to treatment of advanced GC is multimodal with a trend toward perioperative systemic therapy with the importance on neoadjuvant treatment instead of just postoperative therapy especially those who will need a total gastrectomy as postoperative tolerance of chemotherapy is less than $50 \%$. Also, a multimodality team approach to GC care is essential to optimizing patient outcomes which means discussion amongst medical oncologists, surgeons, interventional GI, radiation oncologists, nutritionists, supportive care team as needed. Last but not at least, every patient should be evaluated as an individual and the patient's tumor comprehensively assessed for its phenotypical and genomic characteristics and not just treated as a simple fit into a clinical stage or prognostic category.

FGO: As the Director of GI Minimally Invasive Therapies, could you please give us a brief introduction of current status of GI Minimally Invasive Therapies in the US?

Prof. Woo: Majority of GI cancer operations are performed with the traditional open approach. Safety 


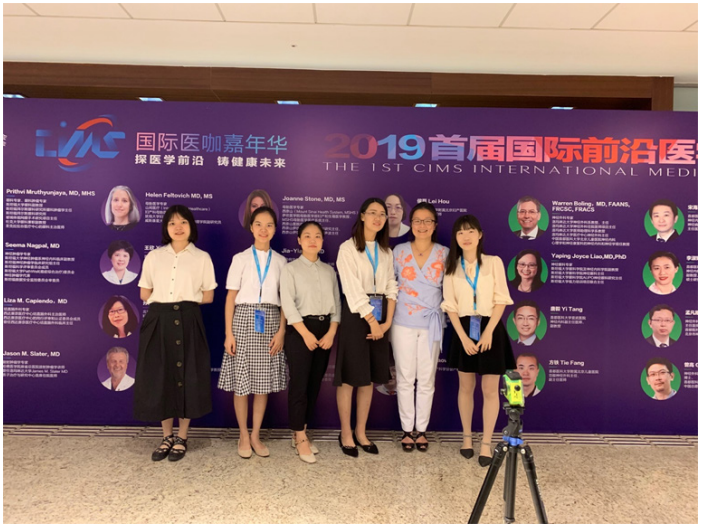

Figure 1 Professor Woo and AME science editors. Left to right: Melissa Zhou, Jessie Bu, Abby Qiu, Angelia Zhou, Yanghee Woo, Crystal Yan.

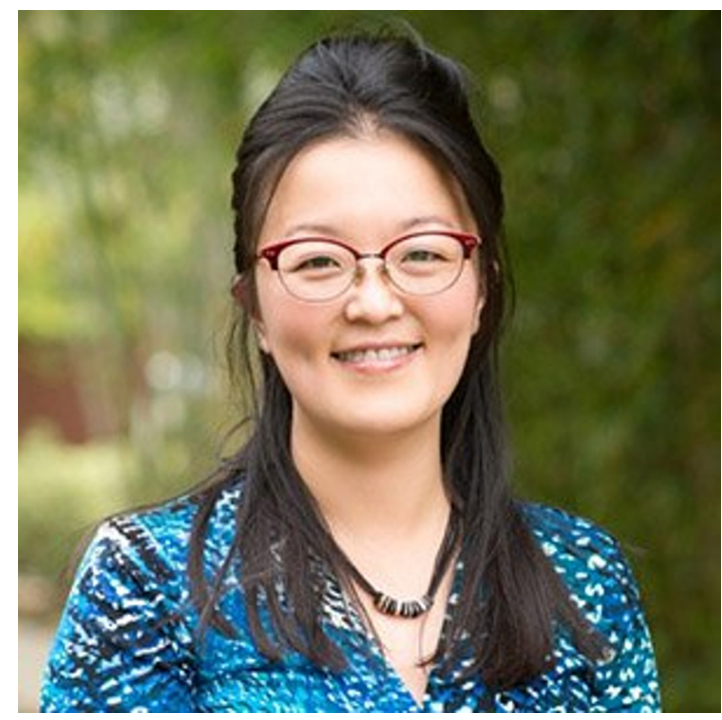

Figure 2 Yanghee Woo, MD.

of GI MIS for has been adopted for less than $20 \%$ of Upper GI/HPB cancer operations. More specifically, while robotic approach for complex abdominal cancers has been pioneered since early 2000, it is just beginning to gain recognition as a safe, feasible and oncologically sound approach for these cancer procedures. The US has experienced a rapid rise in robotic GI cancer operations in the past 5 years but a plateau of the same procedures performed laparoscopically. This highlights the importance of critical evaluation of MIS tools and the development of training programs for surgeons, standardization of procedures, and the optimization of techniques. A well strategized and deliberate integration of new technology and its proper application is necessary to improve GI MIS cancer operations in US and around the world.

\section{FGO: How do you view on the future development of minimally invasive surgery of GC in the coming years?}

Prof. Woo: Robotics and smart technologies will enhance surgeon's ability to perform complex abdominal cancers with greater efficiency and effectiveness.

\section{FGO: It's not easy for women to achieve career success}

Prof. Woo: Yes, but it is true for men. Woman surgeons face unique challenges due to outdated traditions deeply rooted in a male dominant history of surgery and the differing demands of personal life between men and woman. Woman surgeons, however, are achieving not only career success but improving patient outcomes transforming the field of surgical oncology.

FGO: What would be your suggestions for younger female doctors who are devoted to pouring their lives into this field?

Prof. Woo: Being a doctor is easy but becoming the best doctor requires a conviction, dedication and a vision. To achieve success as a world renowned surgeon scientist, it takes a lifetime of sacrifice, passionate pursuits, a lot of hard work, guiding mentors, inspiring colleagues, a bit of luck and endless faith. In addition, we need to challenge the norm, do not accept anything less than excellence. Most importantly the privilege of being a doctor means that we are allowed to love our patients and should constantly give them hope. And remember that as a cancer surgeon, our responsibility is not only to cure the disease but heal the patient.

\section{FGO: Do you think work should come before life?}

Prof. Woo: Why do we think of "work" and "life" as mutually exclusive parts of our lives. Work is a major part of my life and I enjoy work as an inseparable aspect 
of my life. Since I spend most of my awake hours at work like most people, I choose to do something I love at work.

\section{Acknowledgments}

We would like to express our sincerest gratitude to Prof. Yanghee Woo for sharing her insights and expert opinions with us.

Cite this article as: Yan C. Professor Yanghee Woo: globalization of surgical innovation for stomach cancer treatment-a long but exciting road. J Gastrointest Oncol 2020;11(4):829-831. doi: 10.21037/jgo.2019.09.14
Funding: None.

\section{Footnote}

Conflicts of Interest: The author has no conflicts of interest to declare.

(Science Editor: Crystal Yan, JGO, jgo@amepc.org) 